\title{
Fuzzy Comprehensive Evaluation of the Ship Manning
}

\author{
Yu Qian \\ School of Navigation, Wuhan University of Technology \\ Hubei Key Laboratory of Inland Shipping Technology \\ Wuhan, P. R. China \\ yq21cn@126.com \\ Ji Hanqing \\ School of Navigation, Wuhan University of Technology \\ Wuhan, P. R. China \\ sanweiqingniao@163.com
}

\author{
Ma Yong* \\ School of Navigation, Wuhan University of Technology \\ Hubei Key Laboratory of Inland Shipping Technology \\ Wuhan, P. R. China \\ myongdl@163.com \\ * Corresponding Author
}

\begin{abstract}
The ship manning is related to generous factors. That quantifying the factors and building the quantitative comprehensive model of safe levels of the ship manning via the fuzzy comprehensive evaluation can provide positive evaluation standards, which can be referenced for shipping companies and maritime regulators to regulate the ship manning.
\end{abstract}

Keywords- fuzzy comprehensive evaluation; safe level; ship manning; quantitative comprehensive model; evaluation standards.

\section{INTRODUCTION}

On July 19th, 2013, 11 sailors were found guilty of killing the other 22 sailors on a fishing ship (LURONGYU2682) sailing on the Pacific Ocean. This appalling murder at sea reflects many current problems of the vessels manning regulatory system.

SOLAS Chapter v (Safety of Navigation) stipulated in Article 13: contracting governments assume the obligations - when necessary, take measures - to guarantee that each native vessel shall be equipped with enough qualified crew for the life safety at sea [1].

Maritime Traffic Safety Law of the People's Republic of China stipulated in Article 6: the vessel shall be equipped with qualified crew in accordance with the standards to guarantee the vessel safety [2].

Rules of the People's Republic of China on the Administration of Vessel Visas stipulated in Article 7: during the voyage, the ship shall be equipped with the crew whose composition and size are above the standards defined in appendix 1, appendix 2 and appendix 3 [3].

Thus, reasonable crew manning includes two factors constitution and size. Aiming at this problem, one evaluation method of ship manning is presented in this paper, which establishes a comprehensive evaluation system to provide positive referable evaluation standards for the ship manning of ship companies and the ship manning regulation of maritime regulators.

\section{THE QUANTITATIVE COMPREHENSIVE MODEL OF SAFE LEVEL OF THE SHIP MANNING}

\section{A. Establishing factor set}

Factor set is a common set that consists of various factors influencing the evaluation object. That is $U=\left\{u_{1}, u_{2}, u_{3}, \ldots, u_{\mathrm{n}}\right\}$, where $u_{i}(i=1,2, \ldots n)$ represents various influential factors of ship manning from both the crew and companies, which can be "Training, Certification"[4], "Sailing skills and experience", "Interpersonal relationship" of the crew and "Reputation", "Labor intensity" of the companies [5].

Thus, the influential factors of ship manning can be divided into "Crew factors" and "Company factors". We can gain "Crew factor" via respective evaluation of each crew member and "Company factors" via comprehensive evaluation of the company. Such factors as "Training, Certification", "Sailing skills and experience", "Reputation of the company", "Labor intensity" make up the first-order fuzzy factor sets. The "Crew factors" and "Company factors" make up the second-order fuzzy factor sets. Then, first-order and second-order fuzzy factor sets make up the third-order fuzzy factor set of "Ship manning" [6].

The schematic diagram is presented in Fig. 1.

\section{B. Establishing a weight set}

The importance of the various factors mentioned above is varied within different environments. And it is difficult to undertake a quantitative analysis of them. So it is necessary to transform the qualitative analysis into quantitative values via fuzzy mathematical method. And the weight of each factor can be distributed according to the empirical method, set-valued statistics and expert advice.

\section{Establishing the evaluation set}

The evaluation set consists of various possible evaluations which the evaluator may make, usually defined as where $V_{i}(i=1,2, \ldots n)$ represents various possible evaluation results. There are three evaluation sets for the evaluation object is divided into three orders. 
The first evaluation set includes 3 evaluation grades of the first-order fuzzy factor sets, such as "Training, Certification" and "Sailing skills and experience" of the crew as well as "Reputation" and "Labor intensity" of the companies. These evaluation grades are Good, Medium and Bad;

The second includes 3 evaluation grades of the secondorder fuzzy factor sets, such as "Crew factors", "Company factors" comprehensive evaluation. They represent the ability of the crew or company within the evaluation time and they are High, Medium and Low.

The third includes 3 evaluation grades of the final evaluation of ship manning. They are Safe, Medium and Dangerous [7].

\section{The fuzzy comprehensive evaluation}

First, we make the single factor evaluation, which can derive the membership degree of the evaluation object to the element of evaluation set. The evaluation matrix is:

$$
R_{i}=\left(\begin{array}{cccc}
r_{11} & r_{12} & \cdots & r_{1 m} \\
r_{21} & r_{22} & \cdots & r_{2 m} \\
\vdots & \vdots & \ddots & \vdots \\
r_{n 1} & r_{n 2} & \cdots & r_{n m}
\end{array}\right)
$$

Therefore, we evaluate each one-order fuzzy factor firstly (such as "training, certification" and "sailing skills and experience" of the crew as well as "reputation" and "management level" of the company). And we can obtain the comprehensive evaluation by using the product of the factor set and evaluation set according to the weight.

However, the single factor evaluation, merely considering the influence of a single factor in the evaluation set, is not comprehensive. So we should take all the factors into account to get a reasonable evaluation result and then conduct the second-order comprehensive evaluation and the third-order comprehensive evaluation to obtain the final evaluation of ship safety, which is the fuzzy comprehensive evaluation. The final evaluation result is

$$
B=A * R=\left(a_{1}, a_{2} \ldots a_{n}\right) *\left(\begin{array}{cccc}
r_{11} & r_{12} & \cdots & r_{1 m} \\
r_{21} & r_{22} & \cdots & r_{2 m} \\
\vdots & \vdots & \ddots & \vdots \\
r_{n 1} & r_{n 2} & \cdots & r_{n m}
\end{array}\right)=\left(b_{1}, b_{2} \ldots b_{m}\right)
$$

where $A$ is the weight vector, $R$ is single factor evaluation matrix, $b_{j}(j=1,2, \ldots, n)$, which is defined as the evaluation index of fuzzy comprehensive evaluation, is the membership degree of the evaluation object to element $j$ in the evaluation set when all the factors have been taken into account [8].

\section{REALIZING THE FUZZY COMPREHENSIVE EVALUATION OF SHIP MANNING.}

\section{A. The establishment of evaluation system}

The various influential factors of the ship manning evaluation form the factor set $U=\left[\begin{array}{lllll}u_{1}(x) & u_{2}(x) & \ldots & u_{n}(x)\end{array}\right]$, where $u_{i}(x)$ is a certain project factor.

$x$ ranges from 0 to 100 , reflecting the safety grade of the factors. Assume that $u_{i}(x)$ is the "training, certification" of the crew. The value of $x$ is given by the evaluator according to the crew information and historical data provided by the shipping company and the last evaluation of the factor.

If the factor is good, $x$ ranges from 65 to 100 . If the factor is medium, $x$ ranges from 35 to 65 . If the factor is bad and threatens the safety of the crew and the ship, $x$ is between 0 and 35 . The evaluation set includes 3 evaluation grades of "training, certification", which can be written as $V=\left\{v_{1} v_{2} v_{3}\right\}$ [9].

\section{B. Establishment of the membership function}

According to the previous description, the value of $x$ represents the safety grade of factors. We can establish a membership function of each evaluated object as follows. According to the correlation of the value of $x$ and the membership degree $r_{i j}\left(r_{i}=\left\{r_{i 1}, r_{i 2}, r_{i 3}\right\}\right)$ of each factor $u_{i}(x)$ to the 3 grades in the evaluation set.

- When $0 \leqslant x<35$

$$
\left\{\begin{array}{l}
r_{i 1}=0 \\
r_{i 2}=1-r_{i 3} \\
r_{i 3}=1 / 70 x+1
\end{array}\right\}
$$

- When $35 \leqslant x \leqslant 50\left\{\begin{array}{l}r_{i 1}=0 \\ r_{i 2}=1 / 30 x-2 / 3 \\ r_{i 3}=1-r_{i 2}\end{array}\right\}$

- When $50<x \leqslant 65\left\{\begin{array}{l}r_{i 1}=1-r_{i 2} \\ r_{i 2}=-1 / 30 x+8 / 3 \\ r_{i 3}=0\end{array}\right\}$

- When $65<x \leqslant 100\left\{\begin{array}{l}r_{i 1}=1 / 70 x-3 / 7 \\ r_{i 2}=1-r_{i 1} \\ r_{i 3}=0\end{array}\right\}$

With a given $x$, we can use the formula to figure out membership degree of a certain factor to each order and determine the relationship matrix $R$ of fuzzy function.

\section{EXAMPLE}

Assume that after the ship manning evaluation within evaluation time limit, the evaluation factor set of "Training, Certification" is $U=(90,82,80,70,75,60,80,70,90)$. And the weight set is $A=(0.2,0.2,0.15,0.1,0.1,0.1,0.05,0.05,0.05)$. From formula (1) - (4) above, we can obtain the evaluation matrix: 


$$
R=\left(\begin{array}{lll}
85.7 & 14.3 & 0 \\
74.3 & 25.7 & 0 \\
71.4 & 27.6 & 0 \\
57.1 & 42.9 & 0 \\
64.3 & 35.7 & 0 \\
33.3 & 66.7 & 0 \\
71.4 & 27.6 & 0 \\
57.1 & 42.9 & 0 \\
85.7 & 14.3 & 0
\end{array}\right)
$$

We can obtain the evaluation set

$$
B=A R=\left(\begin{array}{lll}
\sum_{i=1}^{9} a_{i} r_{i 1} & \sum_{i=1}^{9} a_{i} r_{i 2} & 0
\end{array}\right)=\left(\begin{array}{lll}
69.25 & 30.75 & 0
\end{array}\right)
$$

And normalize it, yielding $B=A * R=\left[\begin{array}{lll}0.69 & 0.31 & 0\end{array}\right]$. Furthermore, we can obtain the evaluation set $V$ via the weighted average method and quantification of the evaluation set. Because the cut-off points of the membership functions is 35,50 and 65 , we obtain $V=\left\{v_{1}, v_{2}, v_{3}\right\}=\{80,85,20\}$ and the evaluation result $V=0.69 * 80+0.31 * 50=70.7>65[10]$.

Therefore, we can conclude the evaluation of "Training, Certification" is good. In the same way, we can obtain the value of $x$ of the "Crew factors" and "Company factors" and the grade of ship manning via repeating the steps above. And the evaluation results can be used as the basis data of ship manning safety grade.

\section{CONCLUSION}

Within our studies, we establish the ship manning model based on the fuzzy comprehensive evaluation of safety grade. The effectiveness of our approach is verified through the examples. And by using our method, it helps improve the scientific and rationality of ship manning standards evidently.

PSC, CHINA MSA and other maritime regulators can assess the vessels Within their jurisdiction periodically according to fuzzy comprehensive evaluation standard.
And maritime regulators can dispose the vessels according to the assessment result. Shipping companies can also assess the vessels owned by them periodically, Through the method of evaluation, and adjust the management of the vessels according to the result. The result should be registered by the shipping companies and maritime regulators in case that maritime regulators can review the result and determine the responsibility of the accident when some shipwreck happen some day. Therefore, this method is optimistic to the ship navigation safety.

\section{ACKNOWLEDGMENT}

This research was financially supported by the National Natural Science Foundation of China (Grant NO.51309186) and the Fundamental Research Funds for Central University (Grant NO.WUT:2013-IV-015).

\section{REFERENCES}

[1] Convention on the Safety of Life at Sea. Chapter v (Safety of Navigation)[Z].1980.

[2] Maritime Traffic Safety Law of the People's Republic of China[Z] 1984.

[3] Rules of the People's Republic of China on the Administration of Vessel Visas[Z].2007.

[4] Xu Bomin; Qin Zhen. A Study of Ship Collision Accident Causes at Sea Analysis of Fatigue[J]. Navigation Of China, 2009, 32 (4) : 116-119.

[5] International Convention on Standards of Training,Certification and Watch keeping for Seafarers.[Z].1984.

[6] Chen Guobiao. Comprehensive Evaluation Of Ship Manning On fatigue And Safety factors[J]. Guangxi Science And Technology Of Communication, 1998, 23(4): 50-54.

[7] Lv Yan. Study of the Watehing Labor Intensity for Ship Operators and Watehing Arrangement[D]. Dalian: Dalian Maritime University, 2009.

[8] QI Shancai. Supervision on the Manning of the Convenient FlagVessels on the Basis of STCW95 Convention[J]. World Shipping, 2005, 8(1): 37-39.

[9] Li Xing. Problems And Countermeasures Of Ship Manning[J]. Navigation Of Tianjin, 2009,2: 32-34.

[10] Zhan Yulong, Yang Zhiyuan. Fuzzy Factor Analysis on Occupational Stress Source of Seamen[J]. Journal Of Nantong Vocational \& Technical Shipping College, 2009. 8(1):49-52. 


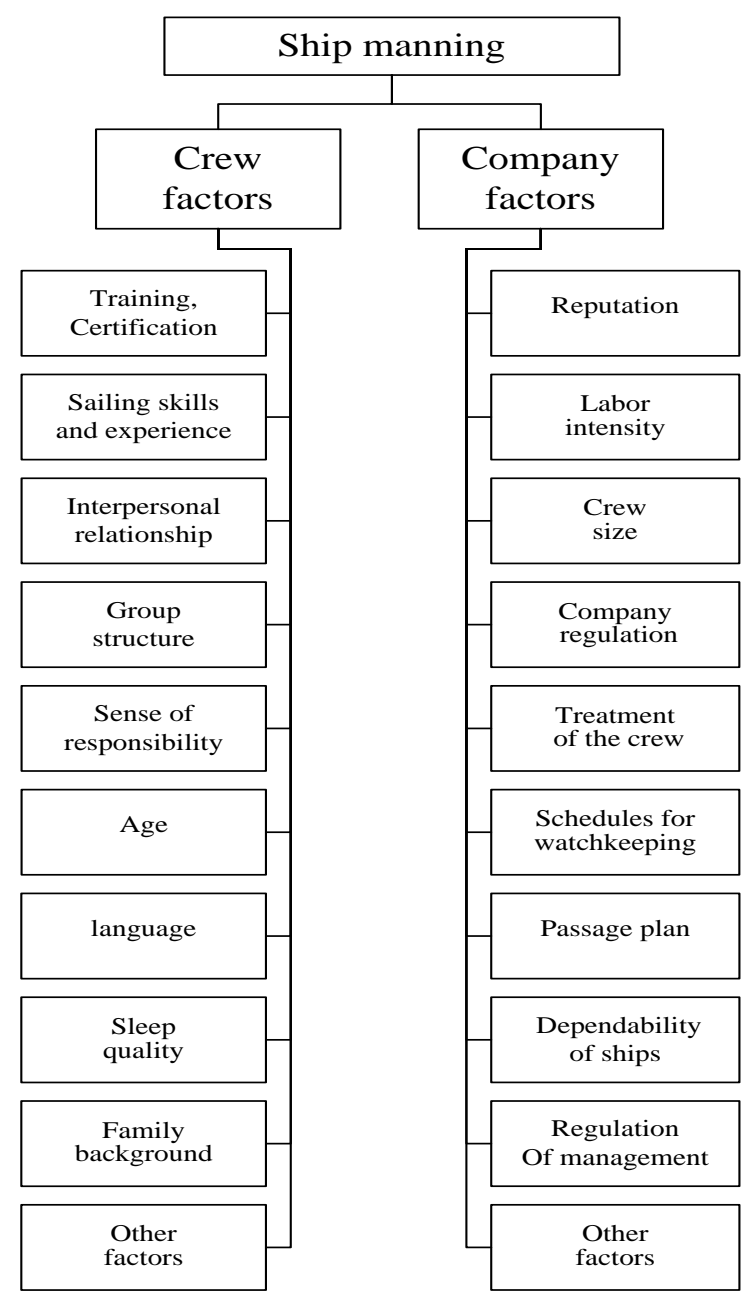

Figure 1. The schematic diagram of the ship manning. 